\title{
Angiographic CT with intravenous administration of contrast medium is a noninvasive option for follow-up after intracranial stenting
}

\author{
Jan-Hendrik Buhk • Paul Lingor • Michael Knauth
}

Received: 28 June 2007 / Accepted: 5 November 2007 / Published online: 2 February 2008

(C) The Author(s) 2007

\begin{abstract}
Intracranial angioplasty and stenting (ICAS) is a therapeutic option in symptomatic intracranial atherosclerotic disease. Adequate follow-up examination is necessary to exclude in-stent restenosis. Conventional intraarterial digital subtraction angiography (ia-DSA) is the current gold standard, but it is an invasive technique and carries the risk of neurological complications. Angiographic CT (ACT) is a new technique that provides a volume dataset of the highest spatial resolution and high contrast resolution derived from a rotational acquisition of a c-arm-mounted flat-panel detector. The feasibility of ACT with intravenous administration of contrast medium (iv-ACT) for follow-up after ICAS is demonstrated. In two patients iv-ACT was performed as a follow-up examination 12 months after ICAS. High-resolution volume data from the rotational acquisitions were processed to provide delineation of the stent lumen as well as imaging of the brain parenchyma and vessels. In both patients the patency of the stent lumen was assessed successfully. In addition, all other brain vessels were displayed in a manner similar to their appearance on $\mathrm{CT}$ angiograms. The brain parenchyma was also adequately imaged in a manner similar to its appearance on CT images. We demonstrated the feasibility and diagnostic value of iv-ACT for follow-up imaging after ICAS. This new application has the potential to become the imaging method
\end{abstract}

\footnotetext{
J.-H. Buhk $(\bowtie) \cdot$ M. Knauth

Department of Neuroradiology, University of Goettingen,

Robert-Koch-Str. 40,

37075 Goettingen, Germany

e-mail: jbuhk@gwdg.de

P. Lingor

Department of Neurology, University of Goettingen,

Goettingen, Germany
}

of choice after ICAS since it not only enables visualization of the patency of the stent lumen but also is minimally invasive and provides additional information about all brain arteries and the brain parenchyma.

Keywords Rotational angiography .

Angiographic computed tomography · Flat-panel .

Cerebrovascular disease $\cdot$ Intracranial stenting

\section{Introduction}

In symptomatic intracranial atherosclerotic disease refractive to medical treatment intracranial angioplasty and stenting (ICAS) is a therapeutic option increasingly used in specialized stroke centers. However, restenosis rates ranging from $8 \%$ to $30 \%$ have been reported, underlining the necessity for regular follow-up examinations to exclude significant restenosis [1-4]. CT angiography (CTA) and MR angiography (MRA) are known to suffer from considerable artifacts that often prevent assessment of the stent lumen. Therefore, current follow-up techniques include transcranial Doppler sonography and conventional intraarterial digital subtraction angiography (ia-DSA). However, diagnostic ia-DSA is an invasive technique with a rate of examination-related neurological complications ranging from $0.5 \%$ to $0.8 \%[5,6]$. In addition, ia-DSA is not offered on an outpatient basis at every medical center so patients have to be admitted for at least one night, significantly increasing the treatment costs. Furthermore, patient compliance with follow-up examinations may be a problem because the ia-DSA procedure is not very comfortable.

The new angiographic systems equipped with flat-panel detectors which allow rotational acquisition of volume data 

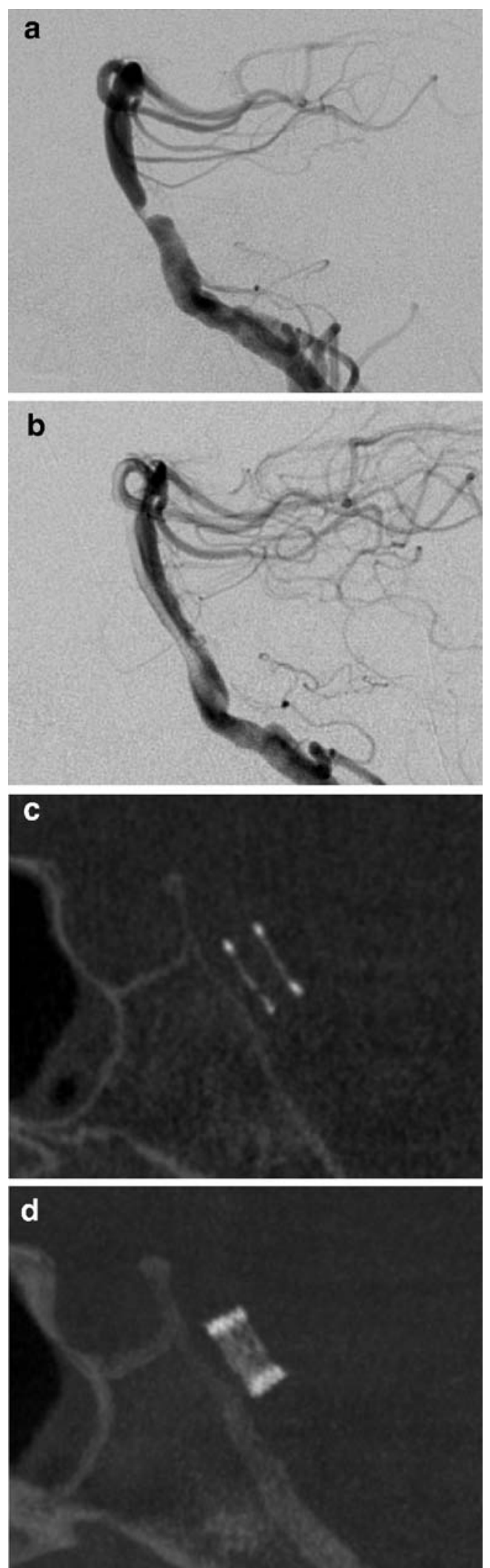

4Fig. 1 Patient 1. a, b DSA with selective injection of contrast medium into the right vertebral artery shows the eccentric high-grade stenosis of the basilar artery (a). After stent placement (b) the lumen of the basilar artery is well reconstructed and considerable inflow from the contralateral vertebral artery can be seen. c, $\mathbf{d}$ Reformatted images in the sagittal plane from an ACT scan directly following the intervention reveal regular deployment of the stent (c MIP, 0.5-mm slice thickness; d MIP, 5-mm slice thickness)
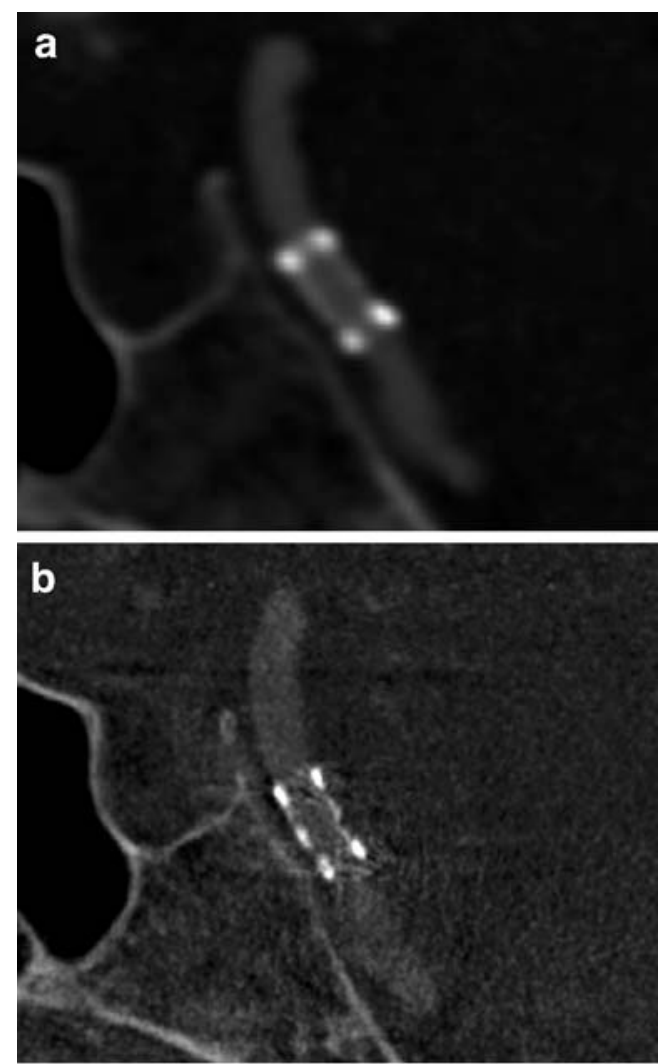

C

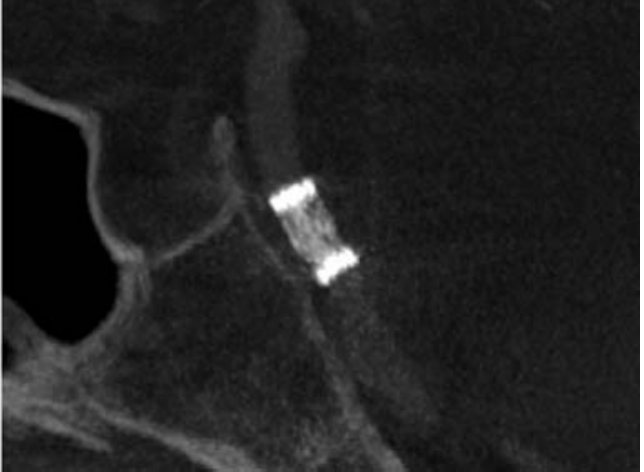

Fig. 2 Patient 1. a CTA image obtained at the 6-month follow-up is not of sufficient quality to reveal the stent lumen, and in particular the proximal and distal markers produce substantial artifacts (MIP, 1-mm slice thickness). b, c iv-ACT images obtained at the 8-month followup show an unchanged configuration of the stent in comparison to the examination performed directly after the intervention (see Fig. 1c,d). The contrast filling is more faint than on the CTA image but the patency of the stent lumen is visible much more clearly on the sagittal cross sections, and the artifact load is acceptable (b MIP, 0.5-mm slice thickness; c MIP, 5-mm slice thickness) 

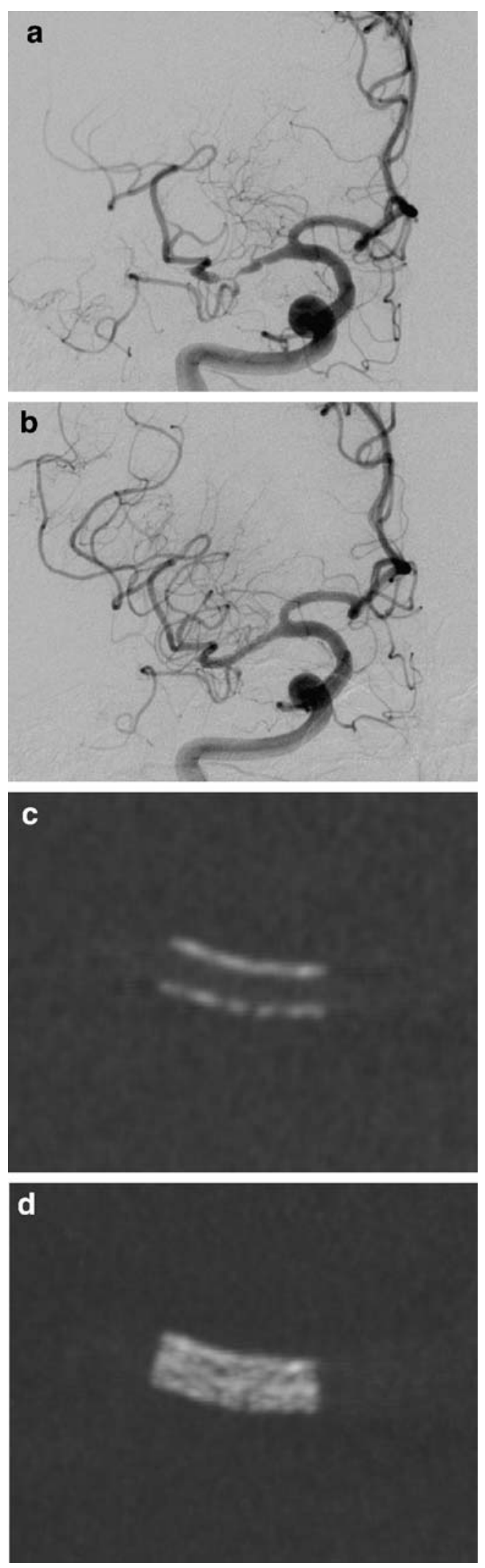

Fig. 3 Patient 2. a, b DSA images with selective injection into the right internal carotid artery reveals a high-grade stenosis of the right middle cerebral artery (MCA) with reduced distal blood flow (a). After stent placement the blood flow is normalized (b). c, d Native ACT reformatted images in paracoronal orientation reveal regular deployment of the stent (c MIP, 0.5-mm slice thickness; d MIP, 5-mm slice thickness)

provide the possibility of CT-like low-contrast imaging [7]. The administration of even highly diluted contrast medium intraarterially gives good delineation of the vessels indicating that intravenous administration of contrast medium could be sufficient. We sought to combine this property with the possibility of nearly artifact-free imaging of smallvessel stents described previously [8].

\section{Methods}

DSA and angiographic CT (ACT) examinations were performed on an AxiomArtis dBA biplane angiography system equipped with flat panel detectors (Siemens Medical Solutions, Forchheim, Germany). The acquisition of rotational datasets was performed with the following parameters $\left(20 \mathrm{~s}-1 \mathrm{k}\right.$ protocol): $20 \mathrm{~s}$ rotation, 538 projections, $220^{\circ}$ total angle, no zoom (detector size $30 \times 40 \mathrm{~cm}$ ), CTDI weighted approximately $22 \mathrm{mGy}$ (manufacturer's information). Postprocessing of the image data to a volume dataset (ACT) was performed on a Leonardo workstation (DynaCT, InSpace 3D software, Siemens). The software includes the application of system-specific filter algorithms in order to correct for beam hardening, radiation scatter, truncated projections and ring artifacts. Postprocessing resulted in volume datasets each defined by a batch of about 400 slices in a $512 \times 512$ matrix. Voxel size was about $0.1 \times 0.1 \times 0.1 \mathrm{~mm}^{3}$. The ACT datasets were further processed to multiplanar reconstructed (MPR) slices with the thin-slice and thickslice maximum intensity projection (MIP) technique parallel and perpendicular to the stent-supplied section of the vessel, respectively. The contrast medium used in the follow-up ACT examination was Imeron 350 (Bracco ALTANA Pharma, Konstanz, Germany). The contrast medium was injected to a volume of $100 \mathrm{ml}$ into a cubital vein at a flow rate of $5 \mathrm{ml} / \mathrm{s}$, and the start delay for rotational acquisition was $20 \mathrm{~s}$. The patient was asked to close his or her eyes and to breathe calmly during the examination.

CTA was performed on a 16-slice CT scanner (Aquilion, Toshiba Medical Systems, Tokyo, Japan) with $0.5-\mathrm{mm}$ slice collimation, intravenous injection of $60 \mathrm{ml}$ Imeron 350 (Bracco ALTANA Pharma), and a CTDI $\mathrm{vol}_{\mathrm{vo}}$ of approximately 50 mGy [9]. Postprocessing was performed on a Vitrea II workstation (Vital Systems, Minnetonka, Minn.). 

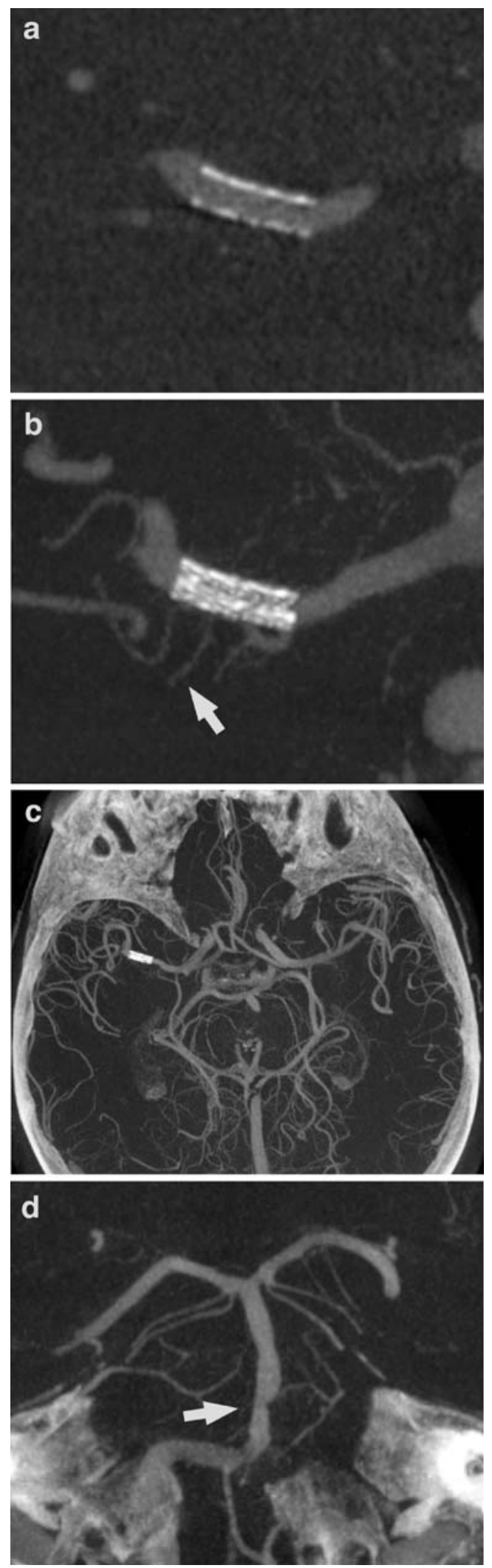

Fig. 4 Patient 2. a, b Iv-ACT images obtained 12 months after the intervention reveal an unchanged stent configuration and full patency of the stent lumen (a MIP, 0.5-mm slice thickness; b MIP, 10-mm slice thickness). Even small perforating arteries originating through the stent struts can be seen (b arrow). c A transverse thick-slice $(30 \mathrm{~mm})$ MIP overview image gives a good impression of the insular MCA branches of both sides. d A second thick-slice $(15 \mathrm{~mm})$ MIP image oriented parallel to the basilar artery reveals a medium-grade stenosis (arrow) and an anatomic variant of the origin of the superior cerebellar artery

\section{Case illustrations}

\section{Case 1}

A 57-year old man suffering from brainstem infarction and recurring transient ischemic attacks of the vertebrobasilar circulation was admitted to our hospital. Transcranial ultrasonography and MRA revealed a high-grade stenosis of the basilar artery. The symptoms occurred despite double platelet inhibition (aspirin and clopidogrel) and ICAS was chosen as therapy. The procedure was performed under general anesthesia. A balloon-mounted Pharos (3.5/8 mm) stent (Micrus Endovascular, Renens, Switzerland) was placed and balloon-inflated to a diameter of $3.2 \mathrm{~mm}$ thereby reconstructing a diameter corresponding to about $90 \%$ of the normal vessel diameter (Fig. 1a,b). The successful deployment of the stent was documented by native ACT (Fig. 1c,d). No complications occurred after the intervention and the patient was discharged 3 days later.

An outpatient follow-up CTA was performed 6 months after the procedure. The stent lumen was not adequately visualized on thin-slice MPR (Fig. 2a). Therefore, admission for follow-up DSA was organized for 2 months later. The patient refused ia-DSA, so ACT with intravenous administration of contrast medium (iv-ACT) was offered as an alternative. Written informed consent for the examination as a novel application of a rotational acquisition was obtained. The examination was successful. Corresponding to the good low-contrast resolution of ACT described previously, the reconstructed images revealed full patency of the stent lumen (Fig. 2b,c) [10]. This result was highly correlated with the flow-rate measured by transcranial Doppler sonography that had remained normal from directly after the intervention until the 8-month follow-up.

\section{Case 2}

An 82-year-old man was admitted suffering from progressive left-sided hemiparesis and impaired consciousness. CTA revealed a high-grade stenosis of the right middle cerebral artery (MCA) and a second middle-grade stenosis of the basilar artery (data not shown). CT-perfusion (CTP) 
showed a prolonged mean transit time in the whole right MCA territory but only small infarcted areas level with the basal ganglia, represented by lowered relative cerebral blood volume. Emergency ICAS was performed under general anesthesia. A balloon-mounted Driver $(2.5 / 8 \mathrm{~mm})$ stent (Medtronic, Minneapolis, Minn.) was successfully placed in the stenosis of the right M1 segment leading to a good morphological result and immediate restoration of normal blood flow (Fig. 3a,b). Proper stent deployment was documented by native ACT (Fig. 3c,d).

The patient was admitted for follow-up DSA 12 months after the procedure. Transcranial Doppler sonography showed a normal flow velocity in the right MCA. This patient also refused ia-DSA. Written informed consent was obtained in advance to perform iv-ACT and to omit ia-DSA if the iv-ACT was of diagnostic quality. The procedure was performed successfully and the reconstructed images revealed full patency of the stent lumen (Fig. 4a,b). All other intracranial arteries were visible at the highest resolution, revealing an unchanged configuration of the medium-grade stenosis of the basilar artery (Fig. 4c,d). The reconstructed brain parenchyma images showed already known residual infarction (data not shown).

\section{Discussion}

In patients suffering from intracranial atherosclerosis, ICAS is a promising new therapeutic option $[1,3,11]$. To identify an in-stent restenosis a reliable imaging technique is needed. Here, DSA is the current gold standard, but it carries a $0.5 \%$ to $0.8 \%$ risk of permanent neurological impairment [5,6]. Additionally, not every center is able to perform ia-DSA on an outpatient basis. The risk may be less with ia-ACT if the injection is performed in the aortic arch. However, this is still an invasive procedure.

In the study reported here we demonstrated that iv-ACT can reliably depict the lumen of small-vessel stents with high spatial resolution. As the low contrast resolution of $\mathrm{ACT}$ is comparable to that of conventional $\mathrm{CT}$, a relatively low concentration of intravascular contrast medium is needed. All intracranial vessels can be viewed simultaneously in a high-quality image. CT-like images of the brain parenchyma are provided as well [10]. It therefore represents a new radiological imaging technique between CTA and ia-DSA.

Even though this new method needs to be evaluated in a larger number of patients, it possibly has many advantages for patients and physicians as a new option in the imaging of cerebrovascular disease: Examinations can be performed on an outpatient basis and the risk of neurological complications is lowered practically to zero. The examination can be performed quickly, in a time similar to that required for a CTA examination. The radiation exposure for the patient from a rotational acquisition is lower than that from a CTA examination (manufacturer's information) and has been shown to be considerably lower than that from a single biplane DSA series [12].

Possible drawbacks of the iv-ACT technique first include movement artifacts. The patient has to lie nearly motionless for about 20 seconds, which requires a high level of compliance and is not always feasible in patients suffering from cerebrovascular disease.

The first stent we examined is a relatively new balloonmounted stent designed for intracranial use (Pharos, Micrus Endovascular, Renens, Switzerland). All dedicated intracranial stents (Neuroform and Wingspan, Boston Scientific, Natick, Mass.; LEO, BALT, Montmorency, France; and Enterprise, Cordis, Miami Lakes, Fl.) have a similar strut size of about $0.06 \mathrm{~mm}$ [13]. In contrast, common coronary stents have thicker struts (e.g. Driver, Medtronic, Minn.: $0.09 \mathrm{~mm}$ ) [14]. Our second patient demonstrates that the lumen of these stents can be visualized as well. This issue is not unimportant because until recently dedicated intracranial stents were not available. Therefore, the majority of stents implanted into intracranial vessels are still coronary stents.

Iv-ACT is a promising new technique for minimally invasive follow-up after intracranial stenting. Other possible applications can be envisaged, such as diagnostic and follow-up imaging before and after endovascular treatment of intracranial aneurysms or arteriovenous malformations.

Conflict of interest statement J.-H. Buhk and M. Knauth have received grants for travel expenses from Siemens Medical Solutions (Forchheim, Germany). P. Lingor declares no conflict of interest.

Open Access This article is distributed under the terms of the Creative Commons Attribution Noncommercial License which permits any noncommercial use, distribution, and reproduction in any medium, provided the original author(s) and source are credited.

\section{References}

1. SSYLVIA Study Investigators (2004) Stenting of Symptomatic Atherosclerotic Lesions in the Vertebral or Intracranial Arteries (SSYLVIA): study results. Stroke 35:1388-1392

2. Wojak JC, Dunlap DC, Hargrave KR, DeAlvare LA, Culbertson HS, Connors J Jr (2006) Intracranial angioplasty and stenting: long-term results from a single center. AJNR Am J Neuroradiol 27:1882-1892

3. Bose A, Hartmann M, Henkes H, Liu HM, Teng MM, Szikora I, Berlis A, Reul J, Yu SC, Forsting M, Lui M, Lim W, Sit SP (2007) A novel, self-expanding, nitinol stent in medically refractory intracranial atherosclerotic stenoses: the Wingspan study. Stroke 38:1531-1537 
4. Jiang WJ, Xu XT, Du B, Dong KH, Jin M, Wang QH, Ma N (2007) Long-term outcome of elective stenting for symptomatic intracranial vertebrobasilar stenosis. Neurology 68:856-858

5. Grzyska U, Freitag J, Zeumer H (1990) Selective cerebral intraarterial DSA. Complication rate and control of risk factors. Neuroradiology 32:296-299

6. Willinsky RA, Taylor SM, TerBrugge K, Farb RI, Tomlinson G, Montanera W (2003) Neurologic complications of cerebral angiography: prospective analysis of 2,899 procedures and review of the literature. Radiology 227:522-528

7. Kalender WA (2003) The use of flat-panel detectors for CT imaging. Radiologe 43:379-387

8. Benndorf G, Strother CM, Claus B, Naeini R, Morsi H, Klucznik R, Mawad ME (2005) Angiographic CT in cerebrovascular stenting. AJNR Am J Neuroradiol 26:1813-1818

9. Van der Molen AJ, Veldkamp WJ, Geleijns J (2007) 16-slice CT: achievable effective doses of common protocols in comparison with recent CT dose surveys. Br J Radiol 80:248-255
10. Heran NS, Song JK, Namba K, Smith W, Niimi Y, Berenstein A (2006) The utility of DynaCT in neuroendovascular procedures. AJNR Am J Neuroradiol 27:330-332

11. Hartmann M, Jansen $O$ (2005) Angioplasty and stenting of intracranial stenosis. Curr Opin Neurol 18:39-45

12. Schueler BA, Kallmes DF, Cloft HJ (2005) 3D cerebral angiography: radiation dose comparison with digital subtraction angiography. AJNR Am J Neuroradiol 26:18981901

13. Canton G, Levy DI, Lasheras JC (2005) Hemodynamic changes due to stent placement in bifurcating intracranial aneurysms. J Neurosurg 103:146-155

14. Mahnken AH, Seyfarth T, Flohr T, Herzog C, Stahl J, Stanzel S, Kuettner A, Wildberger JE, Gunther RW (2005) Flat-panel detector computed tomography for the assessment of coronary artery stents: phantom study in comparison with 16-slice spiral computed tomography. Invest Radiol $40: 8-13$ 